\title{
An equation to predict deaths of nursing home residents during a pandemic
}

\author{
Nursing home residents account for $41 \%$ of all COVID-19 deaths. Understanding why this occurs and the interplay \\ between infectious agent, person, nursing home environment and operations, and public health responses is \\ essential for families and governments when making decisions to protect loved ones and citizens, respectively.
}

\author{
Joseph E. Ibrahim
}

$\Lambda$ pproaching a situation where your decision tips the scales between life and death for a loved one is challenging and fraught with difficulty. The difficult decisions faced by families about nursing home care for their older relatives have been exacerbated by an array of new safety and wellbeing concerns presented by the COVID-19 pandemic. Globally, older people or residents living in nursing homes account for $41 \%$ of all COVID-19 deaths ${ }^{1}$. Many families are now asking themselves, "should our loved one remain in a nursing home, and, if so, is this the right one?", while health practitioners are asking, "which of the nursing homes in our catchment area will have an outbreak of COVID-19 needing substantive assistance or resources, and are we adequately prepared?" The study by Suñer and colleagues'2 investigating risk factors for mortality among nursing homes with COVID-19 in Spain is exciting because it provides valuable empirical data to start addressing these questions.

Their cluster analysis included 167 nursing homes in Catalonia, Spain, with 8,716 long-term care residents of whom 1,629 died, with 1,089 deaths due to COVID-19 between March and June 2020. What makes this study novel is the inclusion of facility-level and geographic populationlevel factors which contribute to mortality. Their use of a unique approach for analysis is creative, conceptually challenging and promotes deeper insights into the cause of mortality and potential interventions. As expected, COVID-19-related and overall mortality was associated with residents who had complex diseases and nursing homes that had lower scores on pandemic preparedness measures, and when the general population dwelling in the same region as the nursing home had a higher incidence of COVID-19. Importantly, Suñer and colleagues describe the potential interactions between infectious agent, person, nursing home environment and operations, and public health and community responses. This contributes valuable insights into the complex interrelationships that determine health outcomes in our modern society (Fig. 1).

The initial assumption was that the large number of deaths in nursing homes was due to the inherent properties of this new virus - that is, a virulent virus equals death. Widely reported was that a person with COVID-19 was tenfold more likely to die than when infected with influenza; that is, a high case fatality rate (proportion of deaths among identified confirmed cases) ${ }^{3}$. Also, an infection with COVID-19 is most dangerous for older people ${ }^{4,5}$, especially those over 80 years of age with chronic disease such as diabetes mellitus, hypertension, heart failure and renal disease, with a case fatality rate in this subpopulation of approximately $15-20 \%$. However, the death rate among older adults in nursing homes was much, much higher than in the general community. Initial case reports from the USA of outbreaks in nursing homes reported fatality rates of residents around $30-35 \%$ (ref. ${ }^{6}$ ), but, as Suñer and colleagues note, large variations in death rates have been observed across long-term care facilities. This suggested there must be something more to the equation than a combination of older persons plus a virulent virus.

The next and most obvious culprit, or variable, to add into the equation to explain this higher mortality rate is the nursing home itself $f^{7,}$. Suñer and colleagues ${ }^{2}$ address the facility-level concept by examining the nursing home's pandemic preparedness using a 12 -item measure. The instrument is very narrowly focused on infection control equipment and tasks related to the availability, use and disposal of personal protective equipment, meaning Suñer and colleagues were not able to fully explore details of a facility's physical building and workforce. Specifically, the building and architecture of a nursing home may contribute to larger infectious outbreak due to shared accommodation (bedrooms and bathrooms), communal spaces for dining and design choices to favour a 'home-like' environment; for example, the removal of hand basins creates impediments to infection control ${ }^{9}$. Suñer et al. used proxy measures to describe the nature of a facility's workforce; however, as they acknowledge, this was lacking specific details. Greater insights require investigating whether higher resident mortality is associated with a facility's workforce leadership, size and clinical skillset. An equation to predict mortality becomes more complex and more accurate by including facility-level variables along with characteristics of the older persons and the virus.

The location of the nursing home and surrounding population was also considered in the study. The authors found that nursing homes in areas with higher COVID-19 14-day incidence had higher mortality rates ${ }^{2}$. Perhaps an explanation for these observations is an inverse relationship between incidence of COVID-19 in a geographic area health and aged care capacity. As the incidence of COVID-19 increases, the capacity of the health system which has already has resource constraints decreases further, leading to rationing and suboptimal care as well as higher mortality. This is supported by other research demonstrating that the adverse impact of the pandemic appears greater on poor and socially disadvantaged populations ${ }^{10}$, and that hospitals performed better (that is, their patients had a lower COVID-19 inpatient mortality rate) when the prevalence of COVID-19 in their surrounding communities was lower ${ }^{11}$. Another relationship worthy of investigating is whether nursing homes within close geographic proximity to a health service have a lower mortality, consistent with the phenomena of better rates of survival observed for persons with acute conditions ${ }^{12}$. 


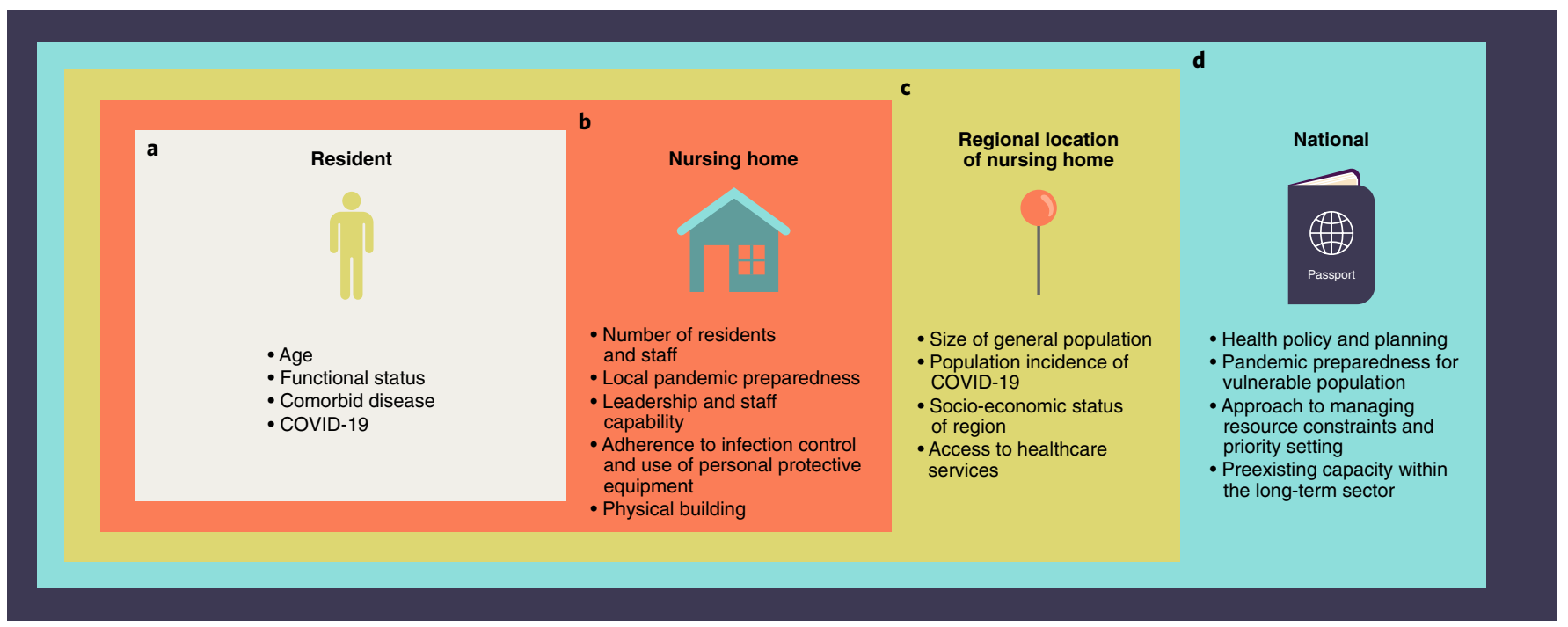

Fig. 1 | Outline of the micro, meso and macro factors that are interacting within and between each level as possible contributing factors to mortality from COVID-19 in nursing homes. a, At the micro level is the interactions between the virus, the person, their functional status and the presence of comorbid diseases. The virulence of the virus contributes to its transmissibility and the nature or severity of illness it causes. The person's age and sex are factors which influence their susceptibility to infection and potential to recover. The person's functional status describes their physiological resilience or frailty, which determines their ability to resist and recover from a severe illness. The presence of comorbid conditions - that is, type and severity of diseases - describes the person's preexisting levels of organ impairment, which increases the risk of complications and mortality. $\mathbf{b}$, At the micro-meso level are the interactions that occur within the nursing home facility which increase or decrease the risk of an outbreak occurring and being contained. It includes the internal population (number of residents and staff), structures (physical building, supplies of personal protective equipment), processes (pandemic preparedness, adherence to infection control) and capacity (leadership, workforce) of a facility. c, At the meso-macro level are the interactions that occur between the nursing home and its local environment. Factors such as the socioeconomic status of the region and the regional population incidence of COVID-19 are likely to contribute to the likelihood of an outbreak occurring in a nursing home. While the size of general population and access to healthcare services in the region will influence the nature of support and resources available to assist the nursing home in responding to a facility-level outbreak. $\mathbf{d}$, At the macro level are the interactions that occur at a national level between the health system and aged care sector. This reflects a nation's capability at the health policy and planning level, the degree to which pandemic preparedness was considered for vulnerable populations and whether there were any preexisting limitations, particularly within the longterm care sector, to respond to. It is also at the macro level that decisions must be made about a nation's approach to managing and setting priorities at times of resource constraints.

The incidence of COVID-19 in the community also reflects how effective the public health interventions are at the regional and national levels. The nation's approach to health policy and planning is often overlooked when investigating the contributing factors to causes of death in an individual. Suñer and colleagues have alluded to and understandably not directly addressed these matters in their study. Sadly, the evidence internationally ${ }^{13,14}$, through public inquiries in multiple countries, identified widespread failures in how the COVID-19 pandemic was managed in nursing homes. These failures led to substantive preventable deaths and human rights abuses in nursing homes. Perhaps the most damning was from the UK: 'As If Expendable 13 exposed gaps in policies and the decision-making processes at all levels of the system. We ought to examine the cascading effects on nursing homes from the successes or failures at national policy level about how each country prepared to respond to the pandemic, and whether there was any specific consideration of the risks and needs of nursing homes. Perhaps what Suñer and colleagues observed could be readily explained through short- and long-term policy and leadership at national and jurisdictional levels, and that we should be looking at the macro rather the micro factors.

The complexity does not end here. While Suñer and colleagues' study included dementia as one of the conditions in the comorbidity count as an individual prognostic factor, persons with dementia face far bleaker health outcomes from the pandemic. In many countries, over half the nursing home population is comprised of persons with dementia ${ }^{15}$. The larger the cohort of persons with dementia in a nursing home, the more difficult it becomes to contain the size of an outbreak. Persons with dementia may have impaired insight, memory and executive function, and this impacts on those around them as well as themselves.
Persons with dementia require more support to adhere to effective infection control measures, such as hand washing, physical distancing and wearing a mask, to contain an outbreak of COVID-19. Even more concerning is the suboptimal care and poorer health outcomes that persons with dementia endure. Along with their cognitive impairment, the existing prepandemic discrimination affecting persons with dementia creates delays in diagnosis due to atypical presentation and lack of knowledge to access health services. Often there is suboptimal adherence to diagnostic testing and treatment when offered, or therapeutic interventions may not be offered at all due to widely held perceptions that the person with dementia has a poor quality of life. Addressing these issues goes well beyond the individual staff member and nursing home facility.

Unravelling the interplay between individual and societal characteristics must continue so we are better prepared for the next pandemic. With this knowledge, we 
are better able to make informed decisions to protect individuals and assist nursing homes. Ultimately, determining if a nursing home is a safe place for an older person to live during a pandemic requires finding the answers to these questions:

1. How prepared and capable is this nursing home in preventing and managing an outbreak?

2. If a resident contracts COVID-19, is this nursing home and the region's acute healthcare service able to provide the clinical care required to optimise survival?

3. If a resident has not contracted COVID-19 during an outbreak in this nursing home, will the nursing home and the region's public health response provide the interventions required to protect individuals from acquiring the infection?

4. If a resident does not contract COVID-19 during an outbreak in this nursing home, will they (i) still receive the personal care to maintain nutrition, hydration and function, and (ii) will the region's health service be able to provide the clinical care required for their preexisting conditions?

5. If a resident has dementia, how will this be accommodated in the response to the preceding questions?
The goal is to identify preventable factors and reduce premature deaths from the pandemic, and the preceding questions help to identify the different layers at play. Premature deaths are prevented by reducing the risk of becoming infected with COVID19 (Q1 and Q3), having prompt access to appropriate clinical therapy to treat the infection (Q2), continuing care for ongoing needs to avoid harm from neglect (Q4i) and clinical care for ongoing chronic diseases or new acute conditions (for example, a fractured hip) unrelated to COVID-19 (Q4bii). Future research should also explore the characteristics, underlying frameworks and trade-offs used to formulate the emergency management pandemic response from the perspective of the individual, nursing home, acute healthcare, public health and community. Perhaps the simpler answer to what is an increasingly complex equation for predicting nursing home mortality is found by asking how well does our country provide for our vulnerable older population in long-term care, and have our health policymakers made concerted and sensible preparations for a pandemic? (Fig. 1).

\section{Joseph E. Ibrahim ${ }^{凶}$}

Health Law and Ageing Research Unit, Department of Forensic Medicine, Monash University, Victorian Institute Forensic Medicine, Southbank, Victoria,
Australia.

凶e-mail: joseph.ibrahim@monash.edu

Published online: 15 July 2021

https://doi.org/10.1038/s43587-021-00083-x

References

1. Comas-Herrera, A. et al. Mortality associated with COVID-19 in care homes: international evidence. LTCCovid https://bit. ly/3fCQ4KY (2021)

2. Suñer, C. et al. Nat. Aging https://doi.org/10.1038/s43587-02100079-7 (2021).

3. Maragakis, L. L. Coronavirus Disease 2019 vs. the Flu. Johns Hopkins Medicine https://bit.ly/3whmkJq (2021).

4. Zhou, F. et al. Lancet 395, 1054-1062 (2020).

5. Guan, W.-J. et al. N. Engl. J. Med. 382, 1708-1720 (2020).

6. McMichael, T. M. et al. N. Engl. J. Med. 382, 2005-2011 (2020).

7. Abrams, H. R., Loomer, L., Gandhi, A. \& Grabowski, D. C. J. Am. Geriatr. Soc. 68, 1653-1656 (2020).

8. Mehta, H. B., Li, S. \& Goodwin, J. S. JAMA Netw. Open 4, e216315 (2021)

9. Anderson, D. C., Grey, T., Kennelly, S. \& O’Neill, D. J. Am. Med. Dir. Assoc. 21, 1519-1524 (2020).

10. Drefahl, S. et al. Nat. Commun. 11, 5097 (2020).

11. Asch, D. A. et al. JAMA Intern. Med. 181, 471-478 (2021).

12. Nicholl, J., West, J., Goodacre, S. \& Turner, J. Emerg. Med. J. 24, 665-668 (2007).

13. United Kingdom: As if Expendable-The UK Government's Failure to Protect Older People in Care Homes During the COVID-19 Pandemic (Amnesty International, 2020); https://www.amnesty. org/en/documents/EUR45/3152/2020/en/

14. Royal Commission Into Aged Care Quality and Safety Aged Care and COVID-19: a Special Report (The Royal Commission into Aged Care Quality and Safety, 2020), https://agedcare.royalcommission.gov.au/publications/ aged-care-and-covid-19-special-report

15. Jain, B., Cheong, E., Bugeja, L. \& Ibrahim, J. J. Am. Med. Dir Assoc. 20, 1558-1565 (2019).

Competing interests

The author declares no competing interests. 